\title{
man \\ Aluminum Doping Effect on Surface Structure of Silver Ultrathin Films
}

\author{
Han Yan ${ }^{1}$, Xiong $\mathrm{Xu}^{1}$, Peng $\mathrm{Li}^{1}{ }^{1 *}$, Peijie $\mathrm{He}^{2}$, Qing Peng ${ }^{3,4,5, *}$ and Can Ding ${ }^{1}$ \\ 1 School of Mechanical and Electronic Engineering, Wuhan University of Technology, Wuhan 430070, China; \\ yanhan@whut.edu.cn (H.Y.); xu22002166@whut.edu.cn (X.X.); dcinwhut@whut.edu.cn (C.D.) \\ 2 School of Power and Mechanical Engineering, Wuhan University, Wuhan 430072, China; hepeijie@whu.edu.cn \\ 3 Physics Department, King Fahd University of Petroleum \& Minerals, Dhahran 31261, Saudi Arabia \\ 4 K.A. CARE Energy Research \& Innovation Center at Dhahran, Dhahran 31261, Saudi Arabia \\ 5 Hydrogen and Energy Storage Center, King Fahd University of Petroleum and Minerals, \\ Dhahran 31261, Saudi Arabia \\ * Correspondence: lp1968@whut.edu.cn (P.L.); qing.peng@kfupm.edu.sa (Q.P.)
}

Citation: Yan, H.; Xu, X.; Li, P.; He, P.; Peng, Q.; Ding, C. Aluminum Doping Effect on Surface Structure of Silver Ultrathin Films. Materials 2022, 15, 648. https://doi.org/10.3390/ ma15020648

Academic Editor: Andrzej Dziedzic

Received: 20 December 2021

Accepted: 11 January 2022

Published: 15 January 2022

Publisher's Note: MDPI stays neutral with regard to jurisdictional claims in published maps and institutional affiliations.

Copyright: (C) 2022 by the authors. Licensee MDPI, Basel, Switzerland. This article is an open access article distributed under the terms and conditions of the Creative Commons Attribution (CC BY) license (https:// creativecommons.org/licenses/by/ $4.0 /)$.

\begin{abstract}
Ultrathin silver films with low loss in the visible and near-infrared spectrum range have been widely used in the fields of metamaterials and optoelectronics. In this study, Al-doped silver films were prepared by the magnetron sputtering method and were characterized by surface morphology, electrical conductivity, and light transmittance analyses. Molecular dynamics simulations and first-principles density functional theory calculations were applied to study the surface morphologies and migration pathway for the formation mechanisms in Al-doped silver films. The results indicate that the migration barrier of silver on a pristine silver surface is commonly lower than that of an Al-doped surface, revealing that the aluminum atoms in the doping site decrease the surface mobility and are conducive to the formation of small islands of silver. When the islands are dense, they coalesce into a single layer, leading to a smoother surface. This might be the reason for the observably lower 3D growth mode of silver on an Al-doped silver surface. Our results with electronic structure insights on the mechanism of the $\mathrm{Al}$ dopants on surface morphologies might benefit the quality control of the silver thin films.
\end{abstract}

Keywords: silver film; surface morphology; magnetron sputtering; molecular dynamics; firstprinciple

\section{Introduction}

Ultrathin metallic films are used for innovative applications, such as flexibility transparent electrodes [1,2], optical metamaterial lenses [3], plasmonic devices [4,5], and lightemitting diodes [6,7]. Due to their advantages in electrical conductivity and low loss in the visible to near-infrared spectrum, ultrathin silver films have attracted a great deal of interest [8]. However, a rough surface with large grain size severely affects the film conductivity and results in additional optical loss. Using conventional deposition techniques for fabricating silver films, including electron beam evaporation and magnetron sputtering, it is difficult to obtain ultrathin and smooth silver film on dielectric substrate surfaces due to the Volmer Weber growth mode during the film deposition process [9].

To address the issue of the roughness of ultrathin silver films, numerous approaches have been applied. A widely used method is to deposit a seed layer before the silver film. Ciesielski et al. investigated the crystallinity and optical parameters of silver layers of 10-35 nm thickness using a 2-10 nm-thick germanium wetting layer pre-deposited on fused silica substrates [10]. Remy et al. prepared $15 \mathrm{~nm}$ silver thin films using a transparent aluminum nitride seed layer in an argon and nitrogen atmosphere. They found that the percolation threshold of silver thin films was reduced by aluminum nitride seeding [11]. Some researchers have provided critical insights into the development of noninvasive 
growth manipulation strategies, in which surface morphology can be promoted by deploying a gaseous species atmosphere. Pliatsikas et al. studied the morphological evolution of magnetron-sputtered silver thin films deposited on weakly interacting silicon dioxide substrates using oxygen as a surfactant. They found that the presence of oxygen throughout all film formation stages leads to smoother film surfaces and larger electrical resistivity of the silver layer [12]. The presence of nitrogen in the gas atmosphere reported by Jamnig et al. revealed similar results [13]. Another improvement in surface roughness was achieved by the co-doping method during silver deposition. Zhang et al. obtained ultrathin and smooth silver films by doping a small amount of aluminum during film deposition, and they found that the thermal stability of Al-doped silver film is significantly improved compared to pure silver [14]. Thomas et al. implemented ultra-thin metallic films in dielectric/metal/dielectric structures using aluminum-doped zinc oxide as the dielectric and an ultrathin aluminum doping silver as the metallic interlayer [15]. In addition to these experiments, there are also many theoretical studies on the surface adsorption of silver [16-19]. Although previous studies have shown that the kinetic energy of the aluminum dopant atom slightly affects the surface morphology of silver films [20], the atomic-scale mechanisms that drive the morphology formation and the evolution of Al-doped silver films are elusive.

In this paper, the roles of aluminum doping in silver film are studied by experiment and first-principles calculations. The surface morphologies for the layer-by-layer growth of silver on an amorphous silicon dioxide surface with aluminum as a dopant are studied by molecular dynamics (MD) simulations. The surface migration behavior of both silver and aluminum on pristine and Al-doped silver surfaces is investigated using first-principles calculations with the frame of Density Functional Theory (DFT). To understand the stabilities of adsorption models, the adsorption energies of silver and aluminum adatoms with different migration paths are systematically examined.

\section{Methods}

\subsection{Experiment Methods}

The samples were prepared using magnetron sputtering equipment (MSP-3200, Beijing Chuangshiweina Technology Co., Ltd., Beijing, China). Polished silica glass substrates were used for the deposition of silver films. The substrates were cleaned using ultrasonic deionized water and then dried using nitrogen gas. The chamber was pumped to an initial pressure of $3 \times 10^{-3} \mathrm{~Pa}$ before the deposition process. The rotating speed of the substrate tray was set to be $20 \mathrm{rpm} / \mathrm{min}$, and no deliberate heating was applied to the substrate during the whole deposition. The metallic films were prepared by the sputtering of silver and aluminum targets at room temperature. The chamber was cooling by circulating water $\left(25^{\circ} \mathrm{C}\right)$. The substrate was attached to a stainless-steel holder with good thermal conduction, and the temperature rise of substrate can be neglected [21,22]. The purity of the sputtering materials was $4 \mathrm{~N}$ for silver and aluminum. The diameter and thickness of the sputtering targets are $75 \mathrm{~mm}$ and $5 \mathrm{~mm}$, respectively. The distance between the sputtering target and substrate was kept at $35 \mathrm{~cm}$. The magnetron sputtering sources were tilted in relation to the axis of the substrate holder. All the depositions were performed in an atmosphere of argon, and the deposition pressure was set as $0.6 \mathrm{~Pa}$ for each film growth process. The silver target was sputtered by radio frequency (RF) power, while the aluminum target was sputtered by direct current (DC) power. The frequency of the RF power supply was $13.56 \mathrm{MHz}$. Based on previous experimental results, when both RF and DC power were set to be $200 \mathrm{~W}$, silver and aluminum were sputtered at an average deposition rate of approximately $6 \AA / \mathrm{s}$ and $0.6 \AA / s$, respectively. The surface morphologies of the samples were tested by atomic force microscopy (AFM, Veeco NanoScope MultiMode, Bruker Co., Ltd., Madison, WI, USA), and Nanoscope 7.30 software (Bruker Co., Ltd., Madison, WI, USA) was used for image analysis. The room temperature sheet resistances measurements of the samples were carried out by a four-point probe system (ST-2258C, Suzhou Jingge Electronic Co., Ltd., No.18 Xinmen Road, Suzhou, China ). The transmittance spectra of the samples were 
measured by UV/VIS/NIR Spectrometer (Lambda 750S, PerkinElmer Inc., Waltham, MA, USA), which is defined as the difference in transmittance between silica substrate with and without silver film.

\subsection{Molecular Dynamics Simulations}

Although the formation and evolution of the rough morphology of metallic film on an oxidized substrate were understood from a thermodynamic point of view [23,24], molecular dynamics simulation can analyze the influencing factors of the surface topography from a different perspective. The effect of aluminum doping on the surface morphology of silver films on amorphous silicon oxide substrate was studied using Large-scale Atomic/Molecular Massively Parallel Simulator (LAMMPS) code [25,26]. The simulation box consisted of a substrate region and deposited film region, and the dimensions were $114.04 \AA$ ( $x$-axis $) \times 85.53 \AA$ ( $y$-axis $) \times 120.00 \AA$ ( $z$-axis $)$. The substrate region was filled with amorphous silicon dioxide; that is, the atoms in the substrate were arranged in a disorderly manner, corresponding to the silica glass substrate in the macro system. The deposition atoms of silver and aluminum were injected from the top of deposited film region. The Tersoff potential [27], embedded-atom method (EAM) potential [28], and Lennard Jones (LJ) potential [29] were used for the Si-O system, metallic atoms, and dynamic interactions between atoms, respectively. The incident direction of deposition atoms in each calculation model was perpendicular to the substrate surface. The initial incidence velocity of each deposition atom was set to $6 \AA$ /ps.

\subsection{First-Principles Calculations}

The adsorption and migration pathway of deposition of a pre-deposited silver surface were studied by first-principles calculations within the frame of density functional theory. The Quantum Espresso package [30,31], version 6.8, was used for the calculations, which has been widely used for the study of atom adsorption and migration [32]. In our simulations, the interactions between ionic cores and valence electrons were described by the projector augmented wave (PAW) potentials [33]. The exchange functional and correlation energy were expressed by Perdew-Burke-Ernzerhof (PBE) [34]. The electronic wave functions were expanded with an energy cutoff of 60 Rydberg. The total energy of each calculation was converged to $1 \times 10^{-5} \mathrm{eV}$. The nonlocal van der Waals interactions corrections are considered [35].

Slab models consisting of a substrate layer and vacuum layer were used for simulations, as shown in Figure 1. To investigate the effect of aluminum doping on the deposition of silver film, a single silver atom is substituted by one aluminum atom to denote the aluminum doping silver surface. To understand the aluminum doping mechanism including different lattice planes, the Ag (111) and Ag (100) surface were investigated.

Three series of paths were considered to reveal the effects of aluminum doping on the preferred adsorption configuration and migration of adatoms. Path 1 represents the migration of adatom from $S_{1}$ to $D_{1}$, as shown in Figure 1a. Path 2 represents the migration of adatom from $S_{2}$ to $D_{2}$. Path 3 represents the migration of adatom from $S_{3}$ to $D_{3}$, as shown in Figure 1c. The adsorption distance was defined as the nearest distance between the adsorbed atom and surface plane, as shown in Figure 1b,d. On each adsorption site, the initial adsorption distance of adatoms above the silver surface was set as $2.5 \AA$. Geometry optimizations were performed for different adsorption sites, in which the bottom substrate layers were fixed at lattice positions, while positions of the adatoms were allowed to relax along the z-axis. The optimized position of the adatom was calculated by minimizing the total energy of the system using the Broyden-Fletcher-Goldfarb-Shanno (BFGS) method [36]. Periodic boundary conditions were applied along the supercell axial directions. The lattice constant of silver unit cell was obtained by lattice optimization, which was $4.0857 \AA$-slightly lower than the reference value of $4.09 \AA$ [37]. 


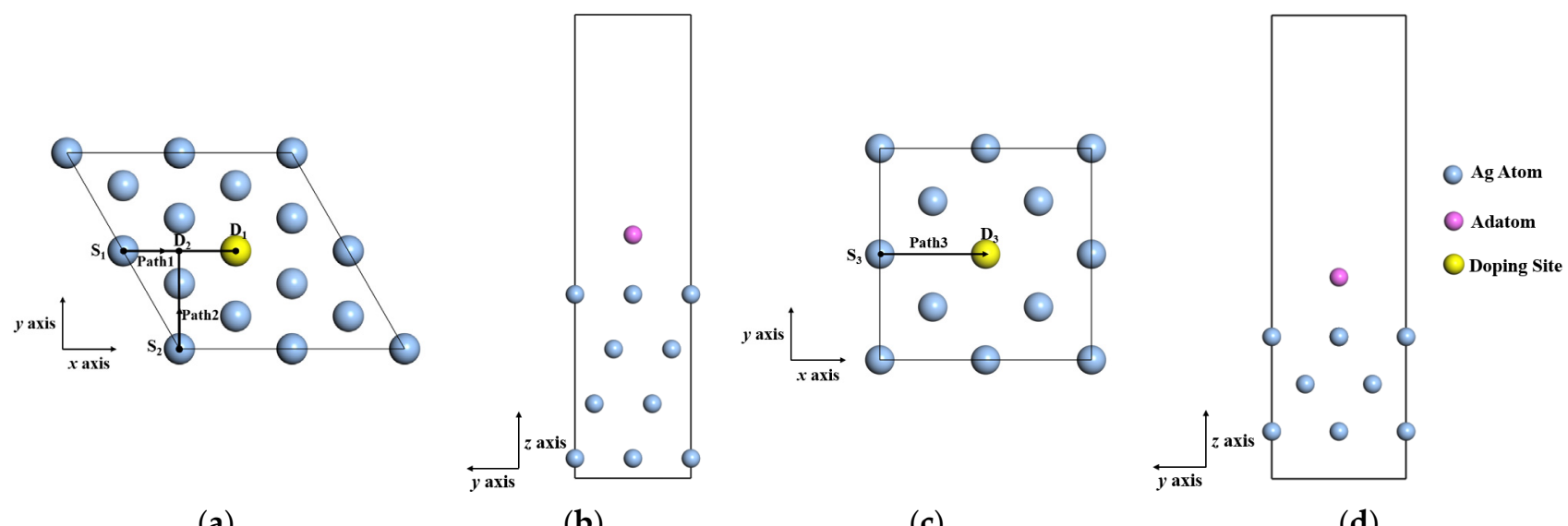

(a)

(b)

(c)

(d)

Figure 1. Schematic illustration of adatom migration on silver surface. (a) Top view of migration paths on $\mathrm{Ag}$ (111) surface, and $\mathrm{D}_{1}$ denotes the doping site; (b) side view of adsorption distance between adatom and $\mathrm{Ag}$ (111) substrate; (c) top view of migration paths on $\mathrm{Ag}(100)$ surface, and $\mathrm{D}_{3}$ denotes the doping site; (d) side view of adsorption distance between adatom and Ag (100) substrate.

The adsorption energy $E_{\mathrm{ad}}$ of an adsorbed atom on silver surface is defined as follows [38]:

$$
E_{\mathrm{ad}}=E_{\text {total }}-E_{\text {adatom }}-E_{\text {substrate }}
$$

where $E_{\text {total }}$ is the total energy of the substrate with the adsorbed atom, $E_{\text {surface }}$ stands for the total energy of the substrate, and $E_{\text {adatom }}$ is the total energy of an isolated adsorbed atom.

\section{Results and Discussion}

\subsection{Experimental Samples Characterization}

Silver thin films with different aluminum components were prepared by magnetron sputtering equipment. The sputtering power of the silver target was kept as constant, while the sputtering power of the aluminum target and total deposition time varied. The deposition parameters are listed in Table 1.

Table 1. Process parameters for each sample.

\begin{tabular}{cccc}
\hline Sample No. & Deposition Time (s) & $\begin{array}{c}\text { Sputtering Power of } \\
\text { Ag (W) }\end{array}$ & $\begin{array}{c}\text { Sputtering Power of } \\
\text { Al (W) }\end{array}$ \\
\hline 1 & 15 & 200 & 0 \\
2 & 15 & 200 & 100 \\
3 & 15 & 200 & 200 \\
4 & 15 & 200 & 300 \\
5 & 9 & 200 & 100 \\
6 & 25 & 200 & 100 \\
\hline
\end{tabular}

The results of the surface morphologies of the samples are shown in Figure 2. The RMS value of silica substrate is $0.726 \mathrm{~nm}$. The undoped silver film shown in Figure 2a has a rough surface and many large protrusions, and therefore, the RMS value of undoped silver film is as large as $9.096 \mathrm{~nm}$. The RMS value of Al-doped silver film is $3.745 \mathrm{~nm}$, which is significantly smaller than that of the undoped silver film. It is obvious that doping silver film with a small amount of aluminum atoms can reduce the surface roughness effectively and promote the formation of a smoother silver film. The surface morphologies of silver films with different aluminum compositions are shown in Figure $2 b-d$. It can be seen that the RMS values of the Al-doped silver film with $100 \mathrm{~W}, 200 \mathrm{~W}$, and $300 \mathrm{~W}$ aluminum target sputtering power are $3.745 \mathrm{~nm}, 3.523 \mathrm{~nm}$, and $13.456 \mathrm{~nm}$, respectively. 
The RMS results are summarized in Figure 3a. It can be seen that the surface roughness can be further improved by increasing the doping concentration of aluminum atoms. However, the surface morphology deteriorates when the doping concentration increases significantly.
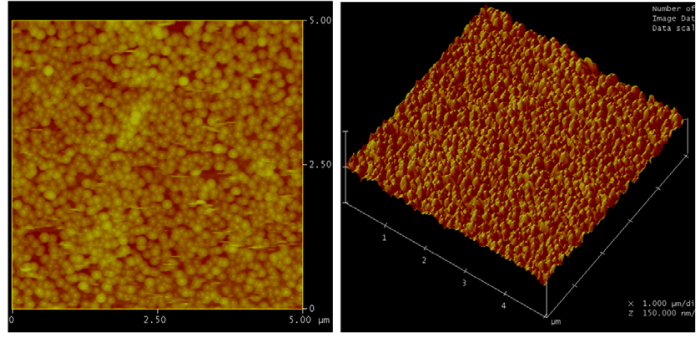

(a)
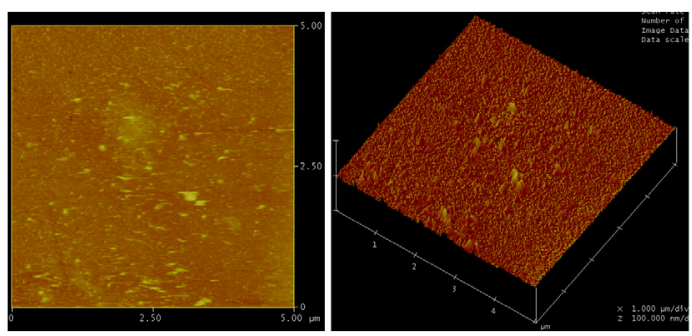

(c)
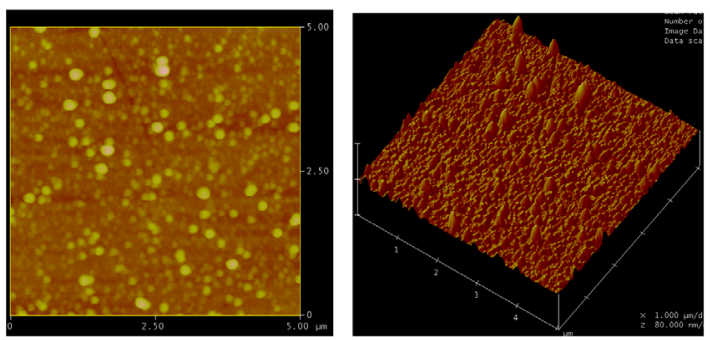

(e)

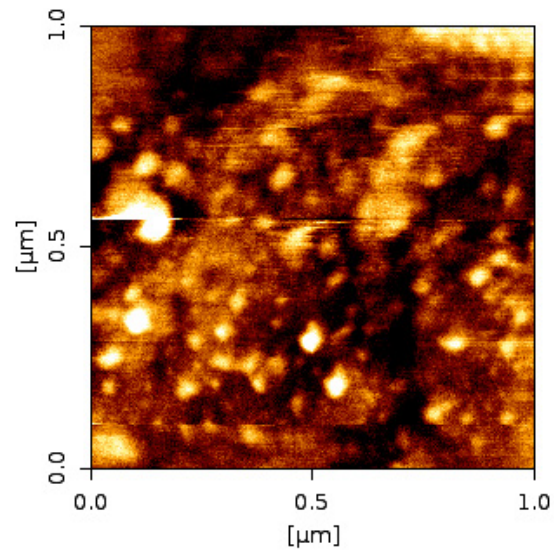

(g)
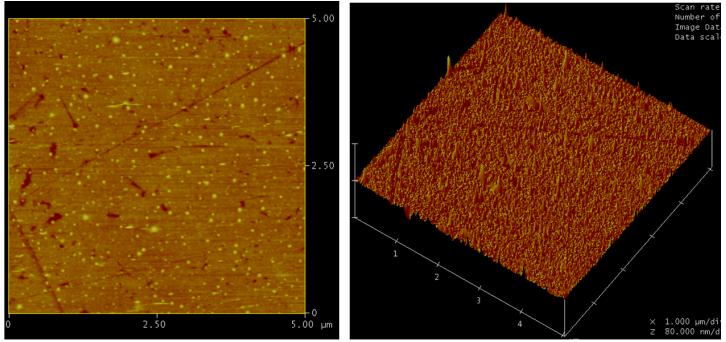

(b)
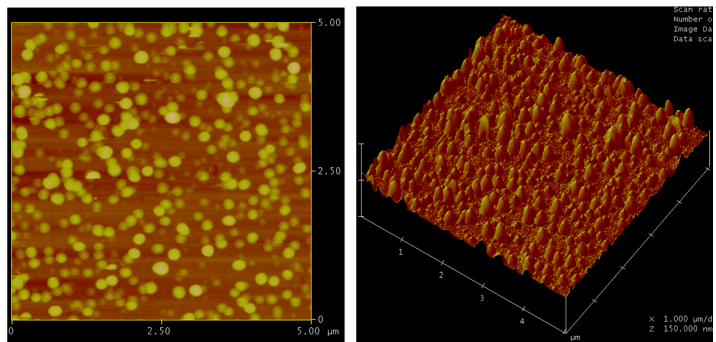

(d)
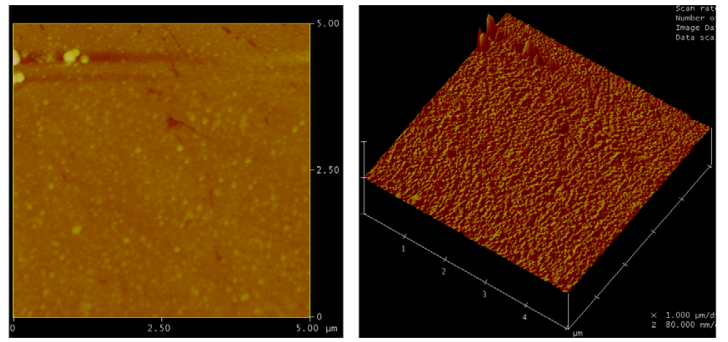

(f)

Figure 2. AFM images of each sample. (a) Sample 1, (b) sample 2, (c) sample 3, (d) sample 4, (e) sample 5, (f) sample 6, (g) substrate. The scan parameters are: scan size is $5.000 \mu \mathrm{m}$, scan rate is $1.001 \mathrm{~Hz}$, number of samples is 256 , image date is height, $\mathrm{x}$ is $1.000 \mu \mathrm{m} / \mathrm{div}$ and $\mathrm{z}$ is varied from 80 to $150 \mathrm{~nm} /$ div. 

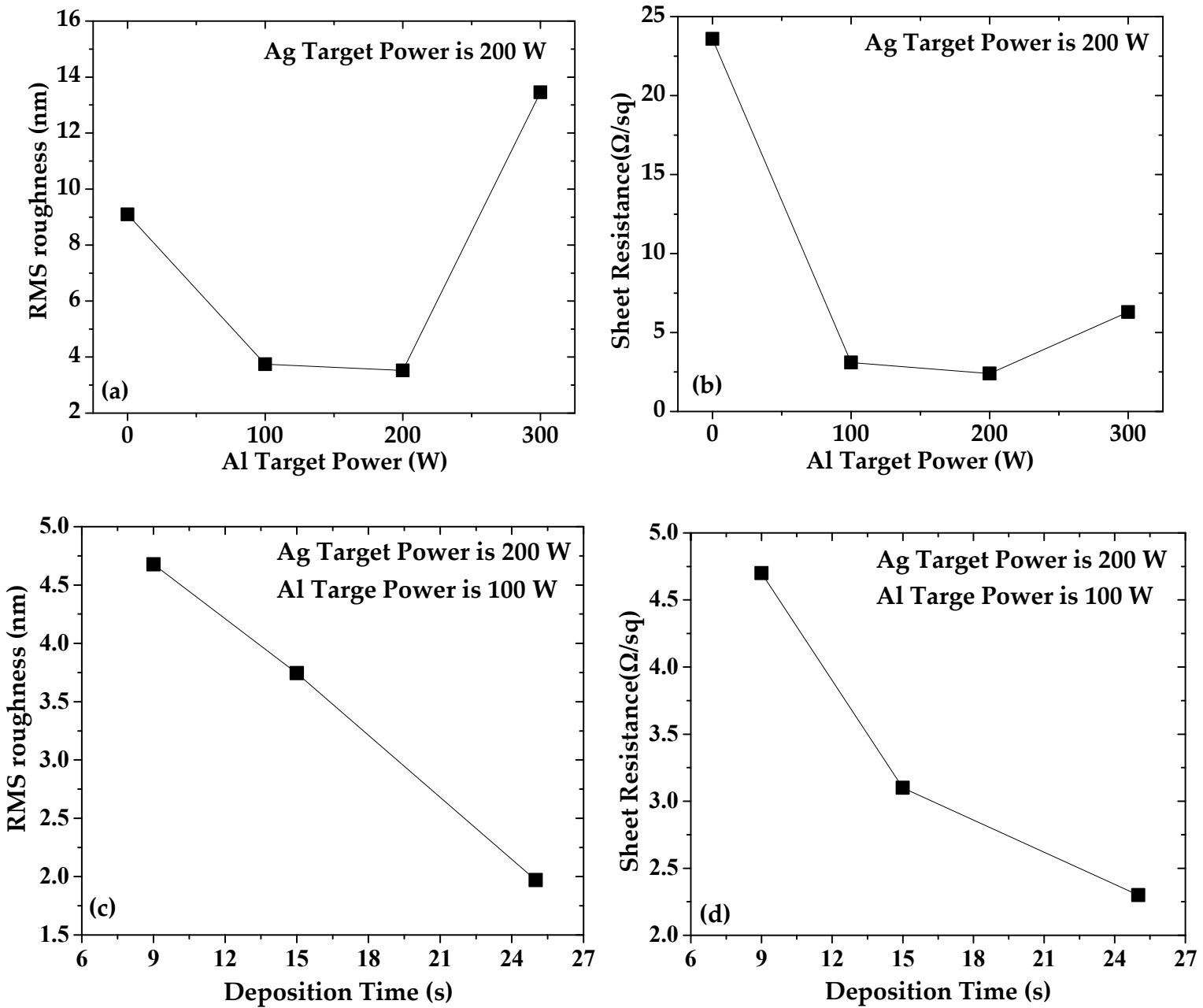

Figure 3. The RMS roughness (a) and sheet resistance (b) of Al-doped silver films under different $\mathrm{Al}$ target power. The RMS roughness (c) and sheet resistance (d) of Al-doped silver films under different deposition time.

The room temperature sheet resistances of silver films were measured using a fourprobe meter. Sample 1 was undoped silver film, and its sheet resistance was $23.6 \Omega / \mathrm{sq}$. Sample 2 was Al-doped silver film with $100 \mathrm{~W}$ aluminum target sputtering power, and its sheet resistance was $3.1 \Omega / \mathrm{sq}$, which is much lower than that of undoped silver film. It is indicated that the degree of surface smoothness has a positive effect on sheet resistance, and that doping a small amount of aluminum atoms not only helps to form smooth silver films but also makes its sheet resistance smaller. The sheet resistance of the Al-doped silver film with aluminum sputtering power of $100 \mathrm{~W}, 200 \mathrm{~W}$, and $300 \mathrm{~W}$ is $3.1 \Omega / \mathrm{sq}, 2.4 \Omega / \mathrm{sq}$, and $6.3 \Omega / \mathrm{sq}$, respectively. The sheet resistance of Al-doped silver film as a function of aluminum target power is shown in Figure $3 \mathrm{~b}$. The results show first a decrease and then an increase with an increase in aluminum composition, which corresponds to the degree of surface smoothness. They reveal that a small amount of aluminum atoms promotes the sheet resistance of Al-doped silver film, and excessive aluminum atoms decrease the sheet resistance.

The surface morphologies of Al-doped silver film with different deposition times were also analyzed. The sputtering powers of silver and aluminum targets were kept constant while the deposition times were set to $9 \mathrm{~s}, 15 \mathrm{~s}$, and $25 \mathrm{~s}$, respectively. The corresponding AFM results are shown in Figure $2 b, e, f$. The size of the surface protrusions gradually decrease as the thickness of Al-doped silver film increases. The surface morphology of the Al-doped silver film will become smoother as its thickness increases. The sheet resistance of 
the Al-doped silver film with deposition times of $9 \mathrm{~s}, 15 \mathrm{~s}$, and $25 \mathrm{~s}$ was $4.7 \Omega / \mathrm{sq}, 3.1 \Omega / \mathrm{sq}$, and $2.3 \Omega$ /sq, respectively. Figure $3 \mathrm{~d}$ shows the sheet resistance of Al-doped silver film as a function of deposition time. It was indicated that the sheet resistance decreases with an increase in thickness.

The transmittance spectra of the Al-doped silver films are shown in Figure 4. It is found that the transmittance of both undoped silver films and Al-doped silver films decreases with the increase in deposition time. The significant drop in transmittance spectra is caused by aluminum doping. The localized plasmon absorption of metal nanoparticles would explain the tendency toward comparatively low optical transmittance $[39,40]$. That is, aluminum doping can affect the particle size and nuclear density of silver nanofilm during deposition. Therefore, the resistivity and transmittance of silver thin films need to be considered together in the application of transparent electrodes.
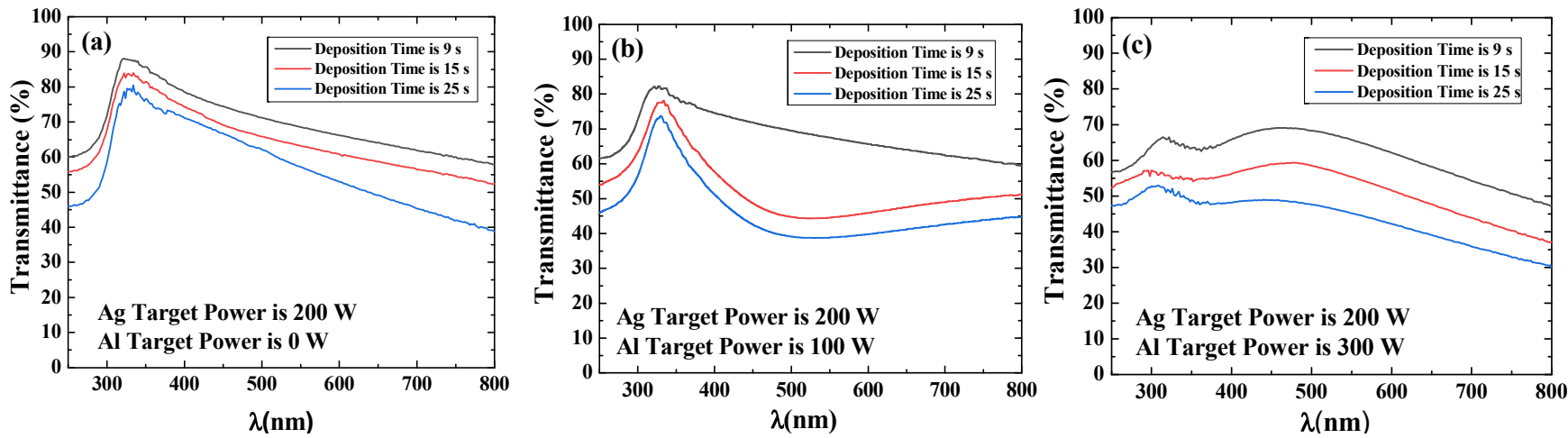

Figure 4. Transmittance results of undoped silver film and Al-doped silver film with different deposition time, and the aluminum target power is (a) $0 \mathrm{~W}$, (b) $100 \mathrm{~W}$ and (c) $300 \mathrm{~W}$, respectively.

\subsection{Simulations on Atomic Structures}

The atomic-scale deposition of aluminum doped silver films on amorphous silicon dioxide substrate with different aluminum compositions and different deposition time were studied by molecular dynamics simulations. The open visualization tool (OVITO) [41] was used to visualize and calculate the surface roughness by root-mean-square (RMS); the results were represented with the Gaussian density method [42] and shown in Figure 5. In order to simulate the effect of aluminum doping on the surface morphology of Al-doped silver film, 20,000 silver atoms were deposited in the system; the number of aluminum atoms varied from 1000 to 8000 to change the doping composition. Figure $5 \mathrm{a}-\mathrm{d}$ show the simulation results of deposition dynamics with varied aluminum composition. The RMS value of Al-doped silver film with an aluminum composition of $5 \%$ and $10 \%$ is $3.296 \AA$ and $2.670 \AA$, respectively. The surface roughness decreases with the increase in aluminum compositions, which is similar to the outcome of varying aluminum target power from 100 to $200 \mathrm{~W}$. The RMS value of Al-doped silver film with aluminum composition of $20 \%$ and $40 \%$ is $3.081 \AA$ and $8.028 \AA$, respectively. The results indicate that excessive aluminum doping increases the surface roughness of Al-doped silver films, which is consistent with the tendency toward variation of our experimental results. 

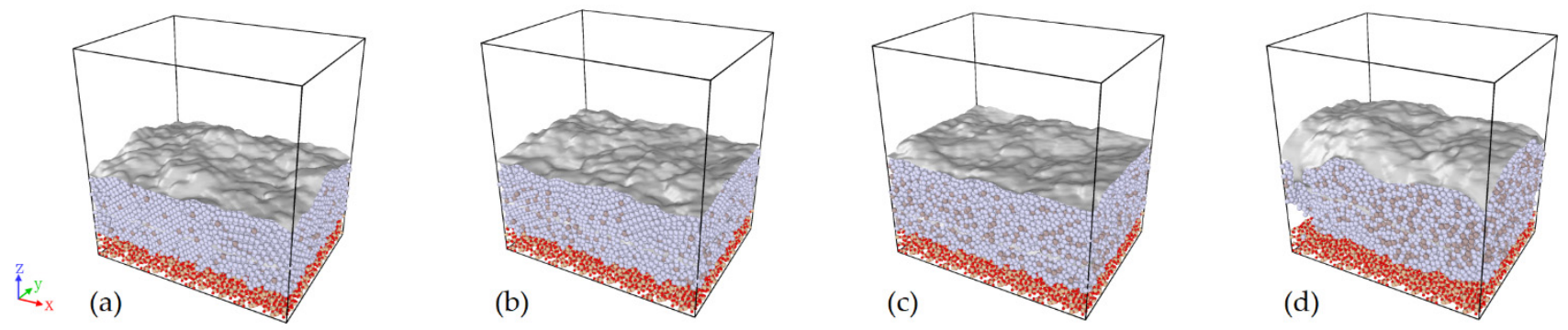

$\mathrm{Ag}$
$\mathrm{Al}$
$\mathrm{O}$
$\mathrm{Si}$

Figure 5. Surface roughness of Al-doped silver films by molecular dynamics simulations. The number of aluminum atoms was set to be (a) 1000, (b) 2000, (c) 4000, and (d) 8000, while the number of silver atoms was kept at 20,000.

The radial distribution function (RDF) of the $\mathrm{Ag}-\mathrm{Ag}$ bond and $\mathrm{Ag}-\mathrm{Al}$ bond of the Al-doped silver film deposited in different aluminum composition are calculated by OVITO and shown in Figure 6. The peak of RDF of the $\mathrm{Ag}-\mathrm{Ag}$ bond that occurs in $2.85 \AA$ with aluminum doping is $5 \%$, which is smaller than the length of the silver bond of $2.889 \AA$ in reference [43]. It is found that the atomic bonds of $\mathrm{Ag}-\mathrm{Ag}$ in 5\% Al-doped silver film are distributed from 2.39 to about $3.5 \AA$ for the nearest neighbors. The minimal pair distance of $\mathrm{Ag}-\mathrm{Ag}$ is reduced to $2.29 \AA$ when the aluminum composition increases to $40 \%$, and the peak position of the $\mathrm{Ag}-\mathrm{Ag}$ pair distance also decreases as the composition of aluminum increases. The results reveal that the incorporation of aluminum makes the nearest neighbor atoms of the silver atoms in the silver film smaller. Figure $6 \mathrm{~b}$ shows that the pair distance of $\mathrm{Ag}-\mathrm{Al}$ remains no matter whether the aluminum composition changes. In our opinion, the nearest neighbor distance of silver-aluminum is almost unaffected by the number of aluminum atoms.
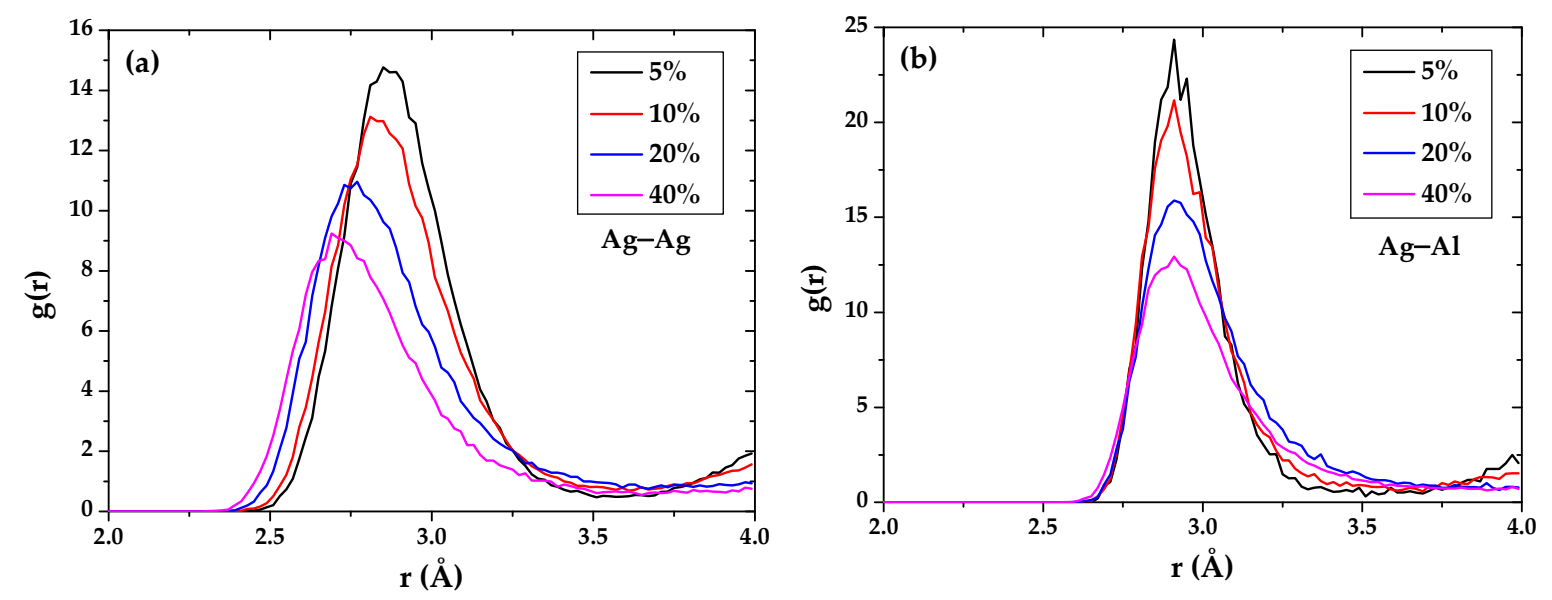

Figure 6. The radial distribution function of (a) $\mathrm{Ag}-\mathrm{Ag}$ and (b) $\mathrm{Ag}-\mathrm{Al}$ in $\mathrm{Al}$-doped silver film deposited in different aluminum composition.

\subsection{First-Principles on Adsorptions and Migration}

To obtain a more detailed description of the atomic origin of aluminum doping effects on the initial deposition on Al-doped silver films, first-principles computational studies of the adsorption and migration of aluminum and silver adatom on a pristine silver and an Al-doped silver surface are presented. The absolute adsorption energies for silver adatom on a pristine Ag (111) and an Al-doped Ag (111) surface are shown in Figure 7. The relatively larger value in absolute adsorption energy indicates the preference of adatom adsorption on the surface. 

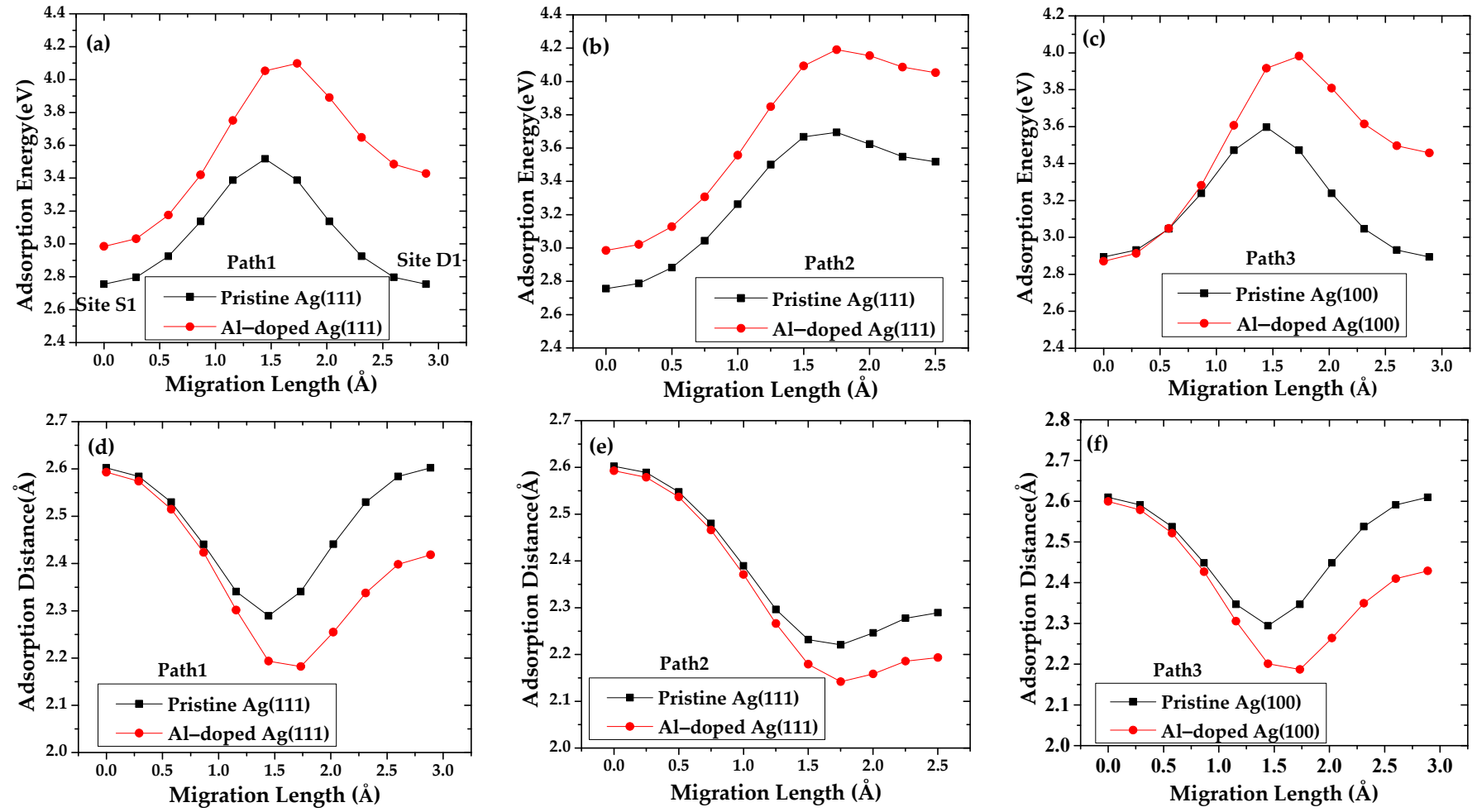

Figure 7. Adsorption energy of silver adatom migration pristine Ag (111)/Ag (100) surface and Al-doped Ag (111)/Ag (100) surface along (a) path 1, (b) path 2 and (c) path 3, and corresponding adsorption distances of silver adatom migration are represented by (d) path 1, (e) path 2 and (f) path 3.

The results show that the adsorption energy of silver on the Al-doped Ag (111) surface along path 1 and path 2 is higher than that of the pristine $\mathrm{Ag}$ (111) surface. Therefore, the silver atoms deposited on the Al-doped Ag (111) surface are more stable. The preferred adsorption position for silver on a pristine $\mathrm{Ag}$ (111) surface along path 1 is the $\mathrm{D}_{2}$ site, which is located in the middle of the bridge between the $S_{1}$ site and $D_{1}$ site, and it is close to the hollow site above the three-fold silver atoms. The maximal adsorption energy and corresponding adsorption distance of silver along path 1 are $3.518 \mathrm{eV}$ and $2.289 \AA$, respectively. In the case that the silver atom at $\mathrm{D}_{1}$ is replaced by an aluminum atom, the maximum adsorption energy and minimum adsorption distance of silver that migrate along path 1 are not at the $\mathrm{D}_{2}$ site but are closer to the $\mathrm{D}_{1}$ site. The corresponding energy and distance are $4.098 \mathrm{eV}$ and $2.182 \AA$, respectively. The calculation results show that the silver adatoms tend to be adsorbed at the site near the aluminum doping atom, the adsorption distance is smaller, and the bond between the adatom and the surface is stronger. For the same reason, the subsequently deposited silver atoms can also be adsorbed in the vicinity of the aluminum atom. In the case that silver migrates along another pathway perpendicular to path 1 , the energetic adsorption position appears in the hollow site, and the corresponding adsorption energy on the pristine $\mathrm{Ag}(111)$ surface is $3.694 \mathrm{eV}$, which is larger than that in the case of path 1 . From the standpoint of the minimum energy of the system, the silver adatom on top of the silver substrate atom is considered to be unstable because it moves toward the bridge site, close to the hollow site. From the crystallographic point of view, the initial stacking growth sequence of the Ag (111) layer continues the FCC stacking of the substrate $[44,45]$; hence, the best adsorption site in the second layer is the hollow position of the first layer.

The maximal adsorption energy along path 2 increased to $4.191 \mathrm{eV}$ with aluminum doping, which revealed that aluminum doping improves the formation of silver adatom deposition in the FCC-stacked sequence. It can be seen from Figure $7 \mathrm{c}$ that as the silver atom 
approaches the aluminum doped atom, the difference between the adsorption energy of the silver adsorbed on the Al-doped Ag (111) surface and pristine Ag (111) surface gradually increases, indicating that the aluminum doping site induces a more stable interaction with the silver adatom. The maximal adsorption energies of silver adsorption on pristine Ag (100) and Al-doped Ag (100) along path3 are $3.597 \mathrm{eV}$ and $3.982 \mathrm{eV}$, respectively. The corresponding adsorption distances of silver on pristine Ag (100) and Al-doped Ag (100) are 2.294 and $2.187 \AA$, respectively. The aluminum-doped atom in the (111) plane has a greater influence on silver atoms than that in the (100) plane.

According to the transition-state theory [46,47], the migration barrier can be defined as the difference between the maximum saddle adsorption energy and minimum valley adsorption energy in the migration path. In our calculations, the migration barriers of silver adsorption on pristine $\mathrm{Ag}$ (111) along path1 is $0.762 \mathrm{eV}$, which is relatively small in comparison with that along path 2 of $0.939 \mathrm{eV}$. The migration barriers of silver adsorption on Al-doped Ag (111) along path 1 and path 2 are $1.113 \mathrm{eV}$ and $1.206 \mathrm{eV}$, respectively. The stronger binding of silver to the Al-doped surface is also reflected in the finding that the migration barrier of silver on pristine $\mathrm{Ag}$ (111) is commonly lower than that of the Al-doped surface. As a consequence, the aluminum atoms in the doping configurations decrease the surface mobility of silver by increasing the diffusion barrier for silver adatoms approaching the aluminum center. Therefore, the presence of substitutional aluminum is conducive to the formation of small islands of silver. If the islands are dense, one expects that they coalesce into a single layer. This might be the reason for the lower $3 \mathrm{D}$ growth mode of silver on an Al-doped Ag (111) surface. The results of silver adsorption on an Ag (111) surface are in agreement with the above standpoint. The migration barrier of silver adsorption on pristine Ag (100) and Al-doped Ag (100) along path 3 is $0.703 \mathrm{eV}$ and $1.110 \mathrm{eV}$, respectively. The calculation results reveal that the presence of aluminum-doped atoms significantly increases the migration barrier of silver atoms for path 3 of the Ag (100) surface.

The Al-doped surface not only affects the adsorption and migration of the silver adatom but also impacts the subsequent deposition of dopant atoms. The calculation results shown in Figure 8 reveal that the surface migration of aluminum adatoms is different from that of silver adatoms. The maximal adsorption energy of aluminum that migrates along path 1 on pristine $\mathrm{Ag}$ (111) and Al-doped Ag (111) is $4.701 \mathrm{eV}$ and $4.620 \mathrm{eV}$, respectively. The corresponding minimum adsorption distance is increased from 2.091 to $2.197 \AA$. In the case that aluminum migrates along path 2 , the maximal adsorption energy of aluminum on pristine $\mathrm{Ag}$ (111) and Al-doped $\mathrm{Ag}$ (111) is $4.879 \mathrm{eV}$ and $4.856 \mathrm{eV}$, respectively. The corresponding minimum adsorption distance is increased from 1.997 to $2.044 \AA$. It is found that the adsorption energy of the $\mathrm{Al}$ adatom at site $\mathrm{S}_{1}$ on a pristine $\mathrm{Ag}(111)$ surface is smaller than that on an Al-doped Ag (111) surface, as the aluminum atoms migrate along path 1 and path 2, and the adsorption energy of the aluminum adatom at the $\mathrm{D}_{1}$ site of pristine Ag (111) becomes lower than that of Al-doped Ag (111) due to aluminum doping.

We also consider the adsorption pathway on Ag (100) surfaces. The maximal adsorption energy of aluminum adsorption on pristine $\mathrm{Ag}(100)$ and Al-doped $\mathrm{Ag}(100)$ is $4.436 \mathrm{eV}$ and $4.594 \mathrm{eV}$, respectively. The corresponding adsorption distance of aluminum on pristine $\mathrm{Ag}(100)$ and Al-doped Ag (100) is 2.073 and $2.177 \AA$, respectively. Aluminum doping increases the adsorption energy of aluminum on the $\mathrm{Ag}(100)$ plane, which suggests that aluminum atoms are more likely to grow epitaxially along another direction. The migration barriers of aluminum adatom on pristine Ag (111) and Al-doped Ag (111) along path 1 are $0.699 \mathrm{eV}$ and $0.704 \mathrm{eV}$, respectively. However, the migration barriers of aluminum adatom on pristine $\mathrm{Ag}$ (111) and Al-doped Ag (111) along path 2 are $0.877 \mathrm{eV}$ and $0.694 \mathrm{eV}$, respectively. The calculation results reveal that the presence of aluminum-doped atoms slightly increases the migration barrier of aluminum atoms for path 1, while it greatly decreases the migration barrier of aluminum atoms along path 2. In the case of the Ag (100) plane, the corresponding value on pristine and Al-doped surface is $0.629 \mathrm{eV}$ and $0.823 \mathrm{eV}$, 
respectively. Aluminum doping significantly reduces the migration length of aluminum atoms on the (100) plane of the silver layer.
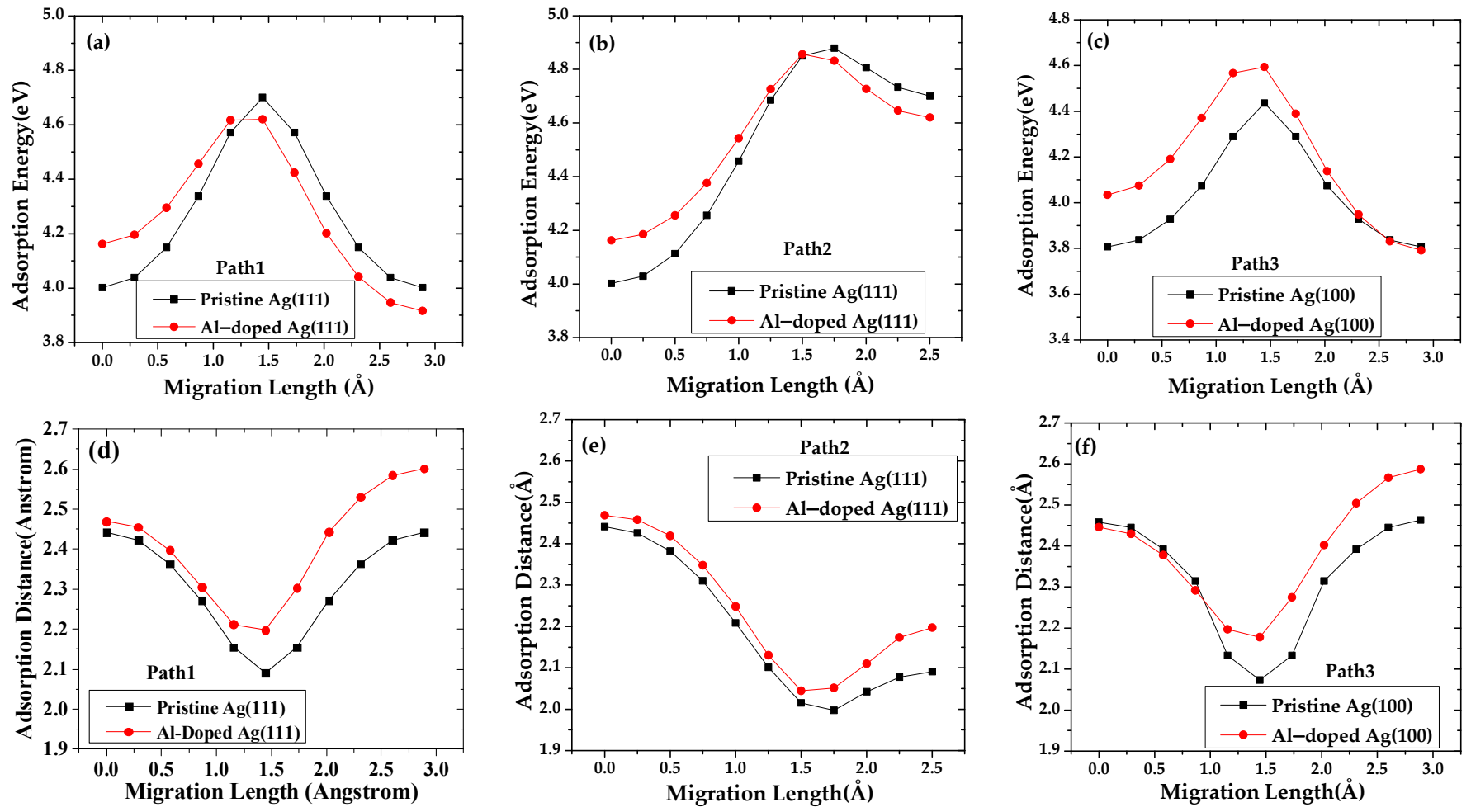

Figure 8. Adsorption energy of aluminum adatom migration pristine Ag (111)/Ag (100) surface and Al-doped Ag (111)/Ag (100) surface along (a) path 1, (b) path 2 and (c) path 3, and corresponding adsorption distances of aluminum adatom migration are represented by (d) path 1, (e) path 2 and (f) path 3.

In a word, the adsorption energy of the aluminum adatom is larger than that of the silver atom at the same adsorption site on the silver surface. The corresponding adsorption distances of aluminum adatoms are lower than those of silver adatoms. It is difficult for silver atoms to move on the surface of silver doped with aluminum, and the surface migration and diffusion length is short. This further explains the fewer three-dimensional silver growth patterns observed on the Al-doped silver surface.

\subsection{Electronic Structures}

Charge density can reveal the charge transition and bonding polarization direction between adatom and surface atoms $[48,49]$. The results are represented by Xcrysden $[50,51]$, as shown in Figure 9. The side view of the iso-surface of charge density mapping projected on the parallel $x-z$ plane containing adatoms along path 1 and the top view of the slice surface between adatoms and the Ag (111) surface are shown. It should be noted that there is an obvious interaction between adatoms and the outermost surface atoms of $\mathrm{Ag}$ (111), while the interaction with the second or inner layer is relatively small.

Compared with the pristine silver interface, the electronic accumulation degree increases around the silver adatom, resulting in improved ionicity nearby. However, the overlap degree of an electron cloud at the interface of aluminum adatom with the doping atoms decreases. The aluminum atoms at the doping position greatly affect the charge distribution between the adsorbed atoms and the substrate. 


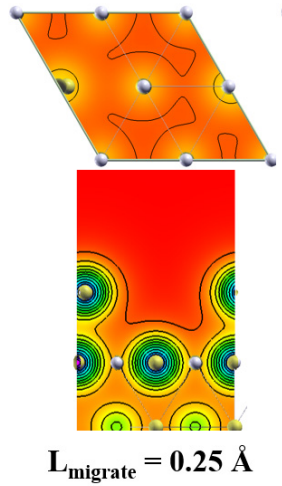

$\mathbf{L}_{\text {migrate }}=0.25 \AA$

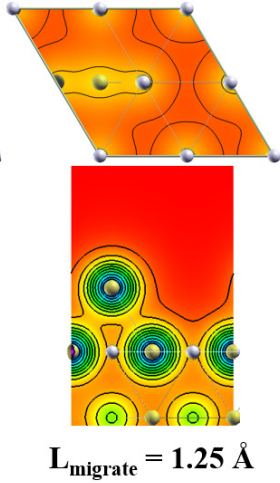

(a)

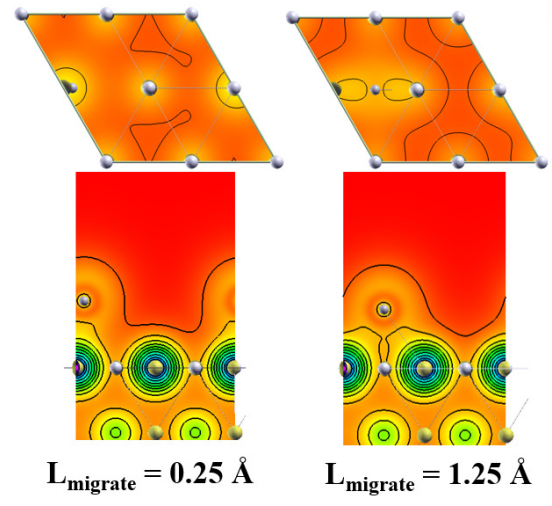

(c)

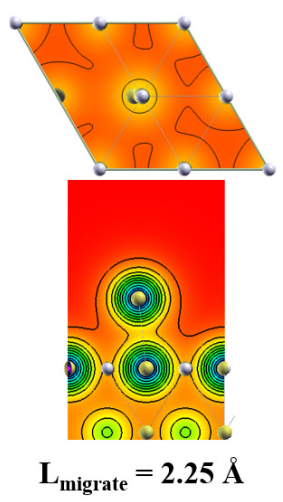

$\mathrm{L}_{\text {migrate }}=\mathbf{2 . 2 5} \AA$

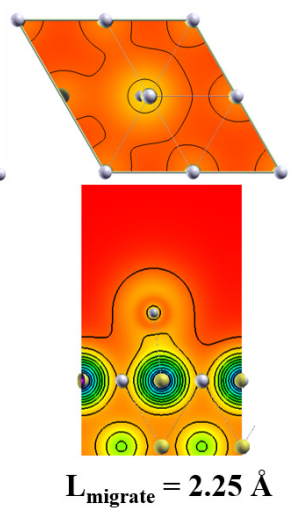

Figure 9. Top and side view of charge density potential energy isolines of adatom adsorption and migration on the $\mathrm{Ag}$ (111) surface along path 1. (a) Silver adatom migration on the pristine $\mathrm{Ag}$ (111) surface, (b) silver adatom migration on the Al-doped $\mathrm{Ag}$ (111) surface, (c) aluminum adatom migration on the pristine $\mathrm{Ag}$ (111) surface, (d) aluminum adatom migration on the Al-doped $\mathrm{Ag}$ (111) surface.

\section{Conclusions}

In this work, the impact of aluminum doping on the surface formation mechanism was studied. The results of fabricating silver nanofilms through an aluminum co-sputtering process revealed that a small amount of aluminum reduces the surface roughness and promotes the sheet resistance of Al-doped silver film, while excessive aluminum deteriorates the surface roughness and decreases the sheet resistances. The theoretical calculation results show that the adsorption energy of silver adatoms on the Al-doped silver surface is higher than that of the pristine silver surface, and silver adatoms tend to be adsorbed at the site near the aluminum doping atom. The aluminum atoms in the doping configurations decrease the surface mobility of silver by increasing the diffusion barrier for silver adatoms approaching the aluminum center. Aluminum doping atoms provide energetically favorable nucleation sites for the incoming silver atoms, which enhances the nuclei density of the silver layer on the surface, leading to an improved surface morphology. Our results might be helpful in surface quality control of the ultrathin silver films.

Author Contributions: Conceptualization, H.Y.; methodology, H.Y., P.L. and Q.P.; software, X.X., C.D. and Q.P.; validation, X.X., P.H. and C.D.; formal analysis, X.X. and C.D.; investigation, H.Y., X.X. and C.D.; data curation, X.X., P.H. and C.D.; writing-original draft preparation, H.Y., X.X. and C.D.; writing-review and editing, P.L. and Q.P.; visualization, X.X. and C.D.; supervision, P.L., Q.P.; funding acquisition, H.Y. and Q.P. All authors have read and agreed to the published version of the manuscript. 
Funding: This research was funded by the National Natural Science Foundation of China, grant number 51775387. Q.P. would like to acknowledge the support provided by the K.A.CARE, Deanship of Scientific Research (DSR) at King Fahd University of Petroleum \& Minerals (KFUPM) through project KACARE211-RFP-02.

Institutional Review Board Statement: Not applicable.

Informed Consent Statement: Not applicable.

Data Availability Statement: The data presented in this study are available on request from the first author and corresponding author.

Conflicts of Interest: The authors declare no conflict of interest.

\section{References}

1. Yun, J. Ultrathin Metal Films for Transparent Electrodes of Flexible Optoelectronic Devices. Adv. Funct. Mater. 2017, $27,1606641$. [CrossRef]

2. Ji, C.; Liu, D.; Zhang, C.; Jay Guo, L. Ultrathin-metal-film-based transparent electrodes with relative transmittance surpassing 100\%. Nat. Commun. 2020, 11, 3367. [CrossRef] [PubMed]

3. Verslegers, L.; Catrysse, P.B.; Yu, Z.; White, J.S.; Barnard, E.S.; Brongersma, M.L.; Fan, S. Planar Lenses Based on Nanoscale Slit Arrays in a Metallic Film. Nano Lett. 2009, 9, 235-238. [CrossRef] [PubMed]

4. $\quad$ Liu, H.; Wang, B.; Leong, E.S.P.; Yang, P.; Zong, Y.; Si, G.Y.; Teng, J.H.; Maier, S.A. Enhanced Surface Plasmon Resonance on a Smooth Silver Film with a Seed Growth Layer. ACS Nano 2010, 4, 3139-3146. [CrossRef] [PubMed]

5. Cazalilla, M.A.; Dolado, J.S.; Rubio, A.; Echenique, P.M. Plasmonic excitations in noble metals: The case of Ag. Phys. Rev. B 2000 61, 8033-8042. [CrossRef]

6. Zhou, S.; Liu, X.; Gao, Y.; Liu, Y.; Liu, M.; Liu, Z.; Gui, C.; Liu, S. Numerical and experimental investigation of GaN-based flip-chip light-emitting diodes with highly reflective Ag/TiW and ITO/DBR Ohmic contacts. Opt. Express 2017, 25, 26615-26627. [CrossRef] [PubMed]

7. Song, M.-G.; Kim, K.-S.; Yang, H.I.; Kim, S.K.; Kim, J.-H.; Han, C.-W.; Choi, H.-C.; Pode, R.; Kwon, J.H. Highly reliable and transparent $\mathrm{Al}$ doped $\mathrm{Ag}$ cathode fabricated using thermal evaporation for transparent OLED applications. Org. Electron. 2020, 76, 105418. [CrossRef]

8. Spinelli, P.; Fuentes Pineda, R.; Scigaj, M.; Ahmad, T.; Wojciechowski, K. Transparent conductive electrodes based on co-sputtered ultra-thin metal layers for semi-transparent perovskites solar cells. Appl. Phys. Lett. 2021, 118, 241110. [CrossRef]

9. Formica, N.; Ghosh, D.S.; Carrilero, A.; Chen, T.L.; Simpson, R.E.; Pruneri, V. Ultrastable and Atomically Smooth Ultrathin Silver Films Grown on a Copper Seed Layer. ACS Appl. Mater. Interfaces 2013, 5, 3048-3053. [CrossRef]

10. Ciesielski, A.; Skowronski, L.; Gorecka, E.; Kierdaszuk, J.; Szoplik, T. Growth model and structure evolution of Ag layers deposited on Ge films. Beilstein J. Nanotechnol. 2018, 9, 66-76. [CrossRef]

11. Ko, R.H.H.; Khalatpour, A.; Clark, J.K.D.; Kherani, N.P. Ultrasmooth ultrathin Ag films by AlN seeding and Ar/N2 sputtering for transparent conductive and heating applications. APL Mater. 2018, 6, 121112. [CrossRef]

12. Pliatsikas, N.; Jamnig, A.; Konpan, M.; Delimitis, A.; Abadias, G.; Sarakinos, K. Manipulation of thin silver film growth on weakly interacting silicon dioxide substrates using oxygen as a surfactant. J. Vac. Sci. Technol. A 2020, 38, 9. [CrossRef]

13. Jamnig, A.; Pliatsikas, N.; Konpan, M.; Lu, J.; Kehagias, T.; Kotanidis, A.N.; Kalfagiannis, N.; Bellas, D.V.; Lidorikis, E.; Kovac, J.; et al. 3D-to-2D morphology manipulation of sputter-deposited nanoscale silver films on weakly interacting substrates via selective nitrogen deployment for multifunctional metal contacts. ACS Appl. Nano Mater. 2020, 3, 4728-4738. [CrossRef]

14. Zhang, C.; Kinsey, N.; Chen, L.; Ji, C.; Xu, M.; Ferrera, M.; Pan, X.; Shalaev, V.M.; Boltasseva, A.; Guo, L.J. high-performance doped silver films: Overcoming fundamental material limits for nanophotonic applications. Adv. Mater. 2017, 29, 1605177. [CrossRef] [PubMed]

15. Tom, T.; Ros, E.; López-Pintó, N.; Miguel Asensi, J.; Andreu, J.; Bertomeu, J.; Puigdollers, J.; Voz, C. Influence of Co-Sputtered Ag:Al Ultra-Thin Layers in Transparent V2O5/Ag:Al/AZO Hole-Selective Electrodes for Silicon Solar Cells. Materials 2020, 13, 4905. [CrossRef]

16. Qin, C.; Sremaniak, L.S.; Whitten, J.L. CO Adsorption on $\mathrm{Ag}(100)$ and $\mathrm{Ag} / \mathrm{MgO}(100)$. J. Phys. Chem. B 2006, 110, 11272-11276. [CrossRef] [PubMed]

17. Mehar, V.; Merte, L.R.; Choi, J.; Shipilin, M.; Lundgren, E.; Weaver, J.F. Adsorption of NO on FeOx Films Grown on Ag(111). J. Phys. Chem. C 2016, 120, 9282-9291. [CrossRef]

18. Chen, B.W.J.; Kirvassilis, D.; Bai, Y.; Mavrikakis, M. Atomic and Molecular Adsorption on Ag(111). J. Phys. Chem. C 2019, 123, 7551-7566. [CrossRef]

19. Salomon, E.; Minissale, M.; Lairado, F.R.; Coussan, S.; Rousselot-Pailley, P.; Dulieu, F.; Angot, T. Pyrene Adsorption on a Ag(111) Surface. J. Phys. Chem. C 2021, 125, 11166-11174. [CrossRef]

20. Tian, Z.; Yan, H.; Peng, Q.; Guo, L.J.; Zhou, S.; Ding, C.; Li, P.; Luo, Q. Atomistic Insights into Aluminum Doping Effect on Surface Roughness of Deposited Ultra-Thin Silver Films. Nanomaterials 2021, 11, 158. [CrossRef] 
21. Liu, W.; Liu, Z.; Yan, F.; Tan, T.; Tian, H. Influence of O2/Ar flow ratio on the structure and optical properties of sputtered hafnium dioxide thin films. Surf. Coat. Technol. 2010, 205, 2120-2125. [CrossRef]

22. Pooyodying, P.; Son, Y.-H.; Sung, Y.-M.; Ok, J.-W. The effect of sputtering Ar gas pressure on optical and electrical properties of flexible ECD device with WO3 electrode deposited by RF magnetron sputtering on ITO/PET substrate. Opt. Mater. 2021, 111829. [CrossRef]

23. Sarakinos, K. A review on morphological evolution of thin metal films on weakly-interacting substrates. Thin Solid Film. 2019, 688, 6. [CrossRef]

24. Campbell, C.T. Metal films and particles on oxide surfaces: Structural, electronic and chemisorptive properties. J. Chem. Soc. Faraday Trans. 1996, 92, 1435-1445. [CrossRef]

25. Favata, A.; Micheletti, A.; Ryu, S.; Pugno, N.M. An analytical benchmark and a mathematica program for MD codes: Testing LAMMPS on the 2nd generation Brenner potential. Comput. Phys. Commun. 2016, 207, 426-431. [CrossRef]

26. Peng, Q.; Meng, F.; Yang, Y.; Lu, C.; Deng, H.; Wang, L.; De, S.; Gao, F. Shockwave generates <100> dislocation loops in bcc iron. Nat. Commun. 2018, 9, 4880. [CrossRef]

27. Munetoh, S.; Motooka, T.; Moriguchi, K.; Shintani, A. Interatomic potential for Si-O systems using Tersoff parameterization. Comput. Mater. Sci. 2007, 39, 334-339. [CrossRef]

28. Wu, H.H.; Trinkle, D.R. Cu/Ag EAM potential optimized for heteroepitaxial diffusion from ab initio data. Comput. Mater. Sci. 2009, 47, 577-583. [CrossRef]

29. Al-Matar, A.K.; Rockstraw, D.A. A generating equation for mixing rules and two new mixing rules for interatomic potential energy parameters. J. Comput. Chem. 2004, 25, 660-668. [CrossRef]

30. Giannozzi, P.; Baroni, S.; Bonini, N.; Calandra, M.; Car, R.; Cavazzoni, C.; Ceresoli, D.; Chiarotti, G.L.; Cococcioni, M.; Dabo, I.; et al. QUANTUM ESPRESSO: A modular and open-source software project for quantum simulations of materials. J. Phys. Condens. Matter 2009, 21, 395502. [CrossRef] [PubMed]

31. Giannozzi, P.; Andreussi, O.; Brumme, T.; Bunau, O.; Buongiorno Nardelli, M.; Calandra, M.; Car, R.; Cavazzoni, C.; Ceresoli, D.; Cococcioni, M.; et al. Advanced capabilities for materials modelling with Quantum ESPRESSO. J. Phys. Condens. Matter 2017, 29, 465901. [CrossRef]

32. Ricard, T.C.; Iyengar, S.S. Efficient and Accurate Approach To Estimate Hybrid Functional and Large Basis-Set Contributions to Condensed-Phase Systems and Molecule-Surface Interactions. J. Chem. Theory Comput. 2020, 16, 4790-4812. [CrossRef]

33. Kresse, G.; Joubert, D. From ultrasoft pseudopotentials to the projector augmented-wave method. Phys. Rev. B 1999, 59, 1758-1775. [CrossRef]

34. Perdew, J.P.; Burke, K.; Ernzerhof, M. Generalized Gradient Approximation Made Simple. Phys. Rev. Lett. 1996, 77, 3865-3868. [CrossRef] [PubMed]

35. Peng, Q.; Wang, G.; Liu, G.-R.; De, S. Van der Waals Density Functional Theory vdW-DFq for Semihard Materials. Crystals 2019, 9 , 243. [CrossRef]

36. Born, D.; Kästner, J. Geometry Optimization in Internal Coordinates Based on Gaussian Process Regression: Comparison of Two Approaches. J. Chem. Theory Comput. 2021, 17, 5955-5967. [CrossRef]

37. Shen, Y.-X.; Kong, L.-T.; Gong, H.-R.; Liu, B.-X. Structural Stability and the Correlation of Lattice Constant versus Tantalum Concentration of the Ag-Based Fcc Solid Solutions Studied by Molecular Dynamics Simulation. Jpn. J. Appl. Phys. 2004, 43, 2589-2593. [CrossRef]

38. Yan, H.; Ku, P.-C.; Gan, Z.-Y.; Liu, S.; Li, P. Strain Effects in Gallium Nitride Adsorption on Defective and Doped Graphene: First-Principles Calculations. Crystals 2018, 8, 58. [CrossRef]

39. Wu, M.; Yu, S.; He, L.; Yang, L.; Zhang, W. High quality transparent conductive Ag-based barium stannate multilayer flexible thin films. Sci. Rep. 2017, 7, 103. [CrossRef]

40. Ye, Y.; Loh, J.Y.Y.; Flood, A.; Fang, C.Y.; Chang, J.; Zhao, R.; Brodersen, P.; Kherani, N.P. Plasmonics of Diffused Silver Nanoparticles in Silver/Nitride Optical Thin Films. Sci. Rep. 2019, 9, 20227. [CrossRef] [PubMed]

41. Stukowski, A. Visualization and analysis of atomistic simulation data with OVITO-the Open Visualization Tool. Model. Simul. Mater. Sci. Eng. 2010, 18, 7. [CrossRef]

42. Krone, M.; Stone, J.; Ertl, T.; Schulten, K. Fast Visualization of Gaussian Density Surfaces for Molecular Dynamics and Particle System Trajectories. In Proceedings of the Eurographics Conference on Visualization, Vienna, Austria, 4-5 June 2012. [CrossRef]

43. Rosalie, J.M.; Bourgeois, L. Silver segregation to $\theta^{\prime}(\mathrm{Al} 2 \mathrm{Cu})-\mathrm{Al}$ interfaces in Al-Cu-Ag alloys. Acta Mater. 2012, 60, 6033-6041. [CrossRef]

44. Howe, C.J.; Cropper, M.D.; Wardle, R.M.; Bailey, P.; Noakes, T.C.Q. Stacking faults in ultra-thin films of silver on Al(111) investigated by medium energy ion scattering. Surf. Sci. 2010, 604, 1658-1665. [CrossRef]

45. Yang, Z.; Zhang, Y.; Zhang, J.; Zhang, K.; Che, J.; Sun, Q. The Adsorption of Ag and Sb on Ag(111) Surface. Phys. Status Solidi B 1999, 212, 281-285. [CrossRef]

46. Govind, N.; Petersen, M.; Fitzgerald, G.; King-Smith, D.; Andzelm, J. A generalized synchronous transit method for transition state location. Comput. Mater. Sci. 2003, 28, 250-258. [CrossRef]

47. Liu, J.; Wen, S.; Wang, Y.; Deng, J.; Chen, X. Transition state search study on the migration of Cu absorbed on the S sites of sphalerite (110) surface. Int. J. Miner. Processing 2016, 147, 28-30. [CrossRef] 
48. Yao, J.-G.; Pan, R.-K.; Jiang, Y.; Yin, D.-F.; Wang, H. Cu segregation at $\alpha$-Al (1 11 1)/ / '-Al3Li (1 11 1) interface. Eur. Phys. J. B 2018, 91, 239. [CrossRef]

49. Sun, S.P.; Li, X.P.; Wang, H.J.; Jiang, Y.; Yi, D.Q. Adsorption of oxygen atom on MoSi2 (110) surface. Appl. Surf. Sci. 2016, 382, 239-248. [CrossRef]

50. Kokalj, A. XCrySDen-A new program for displaying crystalline structures and electron densities. J. Mol. Graph. Model. 1999, 17, 176-179. [CrossRef]

51. Kokalj, A. Computer graphics and graphical user interfaces as tools in simulations of matter at the atomic scale. Comput. Mater. Sci. 2003, 28, 155-168. [CrossRef] 